\title{
Using RDMA Efficiently for Key-Value Services
}

\author{
Anuj Kalia Michael Kaminsky ${ }^{\dagger}$ David G. Andersen \\ Carnegie Mellon University $\dagger^{\dagger}$ Intel Labs \\ \{akalia,dga\}@cs.cmu.edu michael.e.kaminsky@intel.com
}

\begin{abstract}
This paper describes the design and implementation of HERD, a keyvalue system designed to make the best use of an RDMA network. Unlike prior RDMA-based key-value systems, HERD focuses its design on reducing network round trips while using efficient RDMA primitives; the result is substantially lower latency, and throughput that saturates modern, commodity RDMA hardware.

HERD has two unconventional decisions: First, it does not use RDMA reads, despite the allure of operations that bypass the remote CPU entirely. Second, it uses a mix of RDMA and messaging verbs, despite the conventional wisdom that the messaging primitives are slow. A HERD client writes its request into the server's memory; the server computes the reply. This design uses a single round trip for all requests and supports up to 26 million key-value operations per second with $5 \mu$ s average latency. Notably, for small key-value items, our full system throughput is similar to native RDMA read throughput and is over $2 \mathrm{X}$ higher than recent RDMA-based keyvalue systems. We believe that HERD further serves as an effective template for the construction of RDMA-based datacenter services.
\end{abstract}

\section{Keywords}

RDMA; InfiniBand; RoCE; Key-Value Stores

\section{INTRODUCTION}

This paper explores a question that has important implications for the design of modern clustered systems: What is the best method for using RDMA features to support remote hash-table access? To answer this question, we first evaluate the performance that, with sufficient attention to engineering, can be achieved by each of the RDMA communication primitives. Using this understanding, we show how to use an unexpected combination of methods and system architectures to achieve the maximum performance possible on a high-performance RDMA network.

Our work is motivated by the seeming contrast between the fundamental time requirements for cross-node traffic vs. CPU-to-memory lookups, and the designs that have recently emerged that use multiple RDMA (remote direct memory access) reads. On one hand, going between nodes takes roughly 1-3 $\mu \mathrm{s}$, compared to $60-120 \mathrm{~ns}$ for a memory lookup, suggesting that a multiple-RTT design as found in the recent Pilaf [21] and FaRM [8] systems should be fundamentally slower than a single-RTT design. But on the other hand, an RDMA read bypasses many potential sources of overhead, such as servicing interrupts and initiating control transfers, which involve

Permission to make digital or hard copies of all or part of this work for personal or classroom use is granted without fee provided that copies are not made or distributed for profit or commercial advantage and that copies bear this notice and the full citation on the first page. Copyrights for third-party components of this work must be honored. For all other uses. contact the Owner/Author.

Copyright is held by the owner/author(s).

SIGCOMM'14, Aug 17-22 2014, Chicago, IL, USA

ACM 978-1-4503-2836-4/14/08.

http://dx.doi.org/10.1145/2619239.2626299 the host CPU. In this paper, we show that there is a better path to taking advantage of RDMA to achieve high-throughput, low-latency key-value storage.

A challenge for both our and prior work lies in the lack of richness of RDMA operations. An RDMA operation can only read or write a remote memory location. It is not possible to do more sophisticated operations such as dereferencing and following a pointer in remote memory. Recent work in building key-value stores [21, 8] has focused exclusively on using RDMA reads to traverse remote data structures, similar to what would have been done had the structure been in local memory. This approach invariably requires multiple round trips across the network.

Consider an ideal RDMA read-based key-value store (or cache) where each GET request requires only 1 small RDMA read. Designing such a store is as hard as designing a hash-table in which each GET request requires only one random memory lookup. We instead provide a solution to a simpler problem: we design a key-value cache that provides performance similar to that of the ideal cache. However, our design does not use RDMA reads at all.

In this paper, we present HERD, a key-value cache that leverages RDMA features to deliver low latency and high throughput. As we demonstrate later, RDMA reads cannot harness the full performance of the RDMA hardware. In HERD, clients transmit their request to the server's memory using RDMA writes. The server's CPU polls its memory for incoming requests. On receiving a new request, it executes the GET or PUT operation in its local data structures and sends the response back to the client. As RDMA write performance does not scale with the number of outbound connections, the response is sent as a SEND message over a datagram connection.

Our work makes three main contributions:

- A thorough analysis of the performance of RDMA verbs and expose the various design options for key-value systems.

- Evidence that "two-sided" verbs are better than RDMA reads for key-value systems, refuting the previously held assumption $[21,8]$.

- Describing the design and implementation of HERD, a keyvalue cache that offers the maximum possible performance of RDMA hardware.

The following section briefly introduces key-value stores and RDMA, and describes recent efforts in building key-value stores using RDMA. Section 3 discusses the rationale behind our design decisions and demonstrates that messaging verbs are a better choice than RDMA reads for key-value systems. Section 4 discusses the design and implementation of our key-value cache. In Section 5, we evaluate our system on a cluster of 187 nodes and compare it against FaRM [8] and Pilaf [21]. 


\section{BACKGROUND}

This section provides background information on key-value stores and caches, which are at the heart of HERD. We then provide an overview of RDMA, as is relevant for the rest of the paper.

\subsection{Key-Value stores}

DRAM-based key-value stores and caches are widespread in largescale Internet services. They are used both as primary stores (e.g., Redis [4] and RAMCloud [23]), and as caches in front of backend databases (e.g., Memcached [5]). At their most basic level, these systems export a traditional GET/PUT/DELETE interface. Internally, they use a variety of data structures to provide fast, memory-efficient access to their underlying data (e.g., hash table or tree-based indexes).

In this paper, we focus on the communication architecture to support both of these applications; we use a cache implementation for end-to-end validation of our resulting design.

Although recent in-memory object stores have used both tree and hash table-based designs, this paper focuses on hash tables as the basic indexing data structure. Hash table design has a long and rich history, and the particular flavor one chooses depends largely on the desired optimization goals. In recent years, several systems have used advanced hash table designs such as Cuckoo hashing [24, 17, 9] and Hopscotch hashing [12]. Cuckoo hash tables are an attractive choice for building fast key-value systems [9, 31, 17] because, with $K$ hash functions (usually, $K$ is 2 or 3 ), they require only $K$ memory lookups for GET operations, plus an additional pointer dereference if the values are not stored in the table itself. In many workloads, GETs constitute over $95 \%$ of the operations [6, 22]. This property makes cuckoo hashing an attractive backend for an RDMA-based key-value store [21]. Cuckoo and Hopscotch-based designs often emphasize workloads that are read-intensive: PUT operations require moving values within the tables. We evaluate both balanced (50\% PUT/GET) and read-intensive ( $95 \%$ GET) workloads in this paper.

To support both types of workloads without being limited by the performance of currently available data structure options, HERD internally uses a cache data structure that can evict items when it is full. Our focus, however, is on the network communication architecture - our results generalize across both caches and stores, so long as the implementation is fast enough that a high-performance communication architecture is needed. HERD's cache design is based on the recent MICA [18] system that provides both cache and store semantics. MICA's cache mode uses a lossy associative index to map keys to pointers, and stores the values in a circular log that is memory efficient, avoids fragmentation, and does not require expensive garbage collection. This design requires only 2 random memory accesses for both GET and PUT operations.

\subsection{RDMA}

Remote Direct Memory Access (RDMA) allows one computer to directly access the memory of a remote computer without involving the operating system at any host. This enables zero-copy transfers, reducing latency and CPU overhead. In this work, we focus on two types of RDMA-providing interconnects: InfiniBand and RoCE (RDMA over Converged Ethernet). However, we believe that our design is applicable to other RDMA providers such as iWARP, Quadrics, and Myrinet.

InfiniBand is a switched fabric network widely used in highperformance computing systems. RoCE is a relatively new network protocol that allows direct memory access over Ethernet. InfiniBand and RoCE NICs achieve low latency by implementing several layers of the network stack (transport layer through physical layer) in hardware, and by providing RDMA and kernel-bypass. In this section, we provide an overview of RDMA features and terminology that are used in the rest of this paper.

\subsubsection{Comparison with classical Ethernet}

To distinguish from RoCE, we refer to non-RDMA providing Ethernet networks as "classical Ethernet." Unlike classical Ethernet NICs, RDMA NICs (RNICs) provide reliable delivery to applications by employing hardware-based retransmission of lost packets. Further, RNICs provide kernel bypass for all communication. These two factors reduce end-to-end latency as well as the CPU load on the communicating hosts. The typical end-to-end ( $\left.\frac{1}{2} \mathrm{RTT}\right)$ latency in InfiniBand/RoCE is $1 \mu$ s while that in modern classical Ethernet-based solutions [2,18] is $10 \mu \mathrm{s}$. A large portion of this gap arises because of differing emphasis in the NIC design. RDMA is increasing its presence in datacenters as the hardware becomes cheaper [21]. A 40 Gbps ConnectX-3 RNIC from Mellanox costs about \$500, while a 10 Gbps Ethernet adapter costs between $\$ 300$ and $\$ 800$. The introduction of RoCE will further boost RDMA's presence as it will allow sockets applications to run with RDMA applications on the same network.

\subsubsection{Verbs and queue pairs}

Userspace programs access RNICs directly using functions called verbs. There are several types of verbs. Those most relevant to this work are RDMA read (READ), RDMA write (WRITE), SEND, and RECEIVE. Verbs are posted by applications to queues that are maintained inside the RNIC. Queues always exist in pairs: a send queue and a receive queue form a queue pair (QP). Each queue pair has an associated completion queue (CQ), which the RNIC fills in upon completion of verb execution.

The verbs form a semantic definition of the interface provided by the RNIC. There are two types of verbs semantics: memory semantics and channel semantics.

Memory semantics: The RDMA verbs (READ and WRITE) have memory semantics: they specify the remote memory address to operate upon. These verbs are one-sided: the responder's CPU is unaware of the operation. This lack of CPU overhead at the responder makes one-sided verbs attractive. Furthermore, they have the lowest latency and highest throughput among all verbs.

Channel semantics: SEND and RECEIVE (RECV) have channel semantics, i.e., the SEND's payload is written to a remote memory address that is specified by the responder in a pre-posted RECV. An analogy for this would be an unbuffered sockets implementation that required read ( ) to be called before the packet arrived. SEND and RECV are two-sided as the CPU at the responder needs to post a RECV in order for an incoming SEND to be processed. Unlike the memory verbs, the responder's CPU is involved. Two-sided verbs also have slightly higher latency and lower throughput than one sided verbs and have been regarded unfavorably for designing key-value systems [21, 8].

Although SEND and RECV verbs are technically RDMA verbs, we distinguish them from READ and WRITE. We refer to READ and WRITE as RDMA verbs, and refer to SEND and RECV as messaging verbs.

Verbs are usually posted to the send queue of a QP (except RECV, which is posted to the receive queue). To post a verb to the RNIC, an application calls into the userland RDMA driver. Then, the driver prepares a Work Queue Element (WQE) in the host's memory and 


\begin{tabular}{llll} 
Verb & RC & UC & UD \\
\hline SEND/RECV & $\checkmark$ & $\checkmark$ & $\checkmark$ \\
WRITE & $\checkmark$ & $\checkmark$ & $x$ \\
READ & $\checkmark$ & $x$ & $x$
\end{tabular}

Table 1: Operations supported by each connection type. UC does not support READs, and UD does not support RDMA at all.

rings a doorbell on the RNIC via Programmed IO (PIO). For ConnectX and newer RNICs, the doorbell contains the entire WQE [27]. For WRITE and SEND verbs, the WQE is associated with a payload that needs to be sent to the remote host. A payload up to the maximum PIO size (256 in our setup) can be inlined in the WQE, otherwise it can be fetched by the RNIC via a DMA read. An inlined post involves no DMA operations, reducing latency and increasing throughput for small payloads.

When the RNIC completes the network steps associated with the verb, it pushes a completion event to the queue pair's associated completion queue (CQ) via a DMA write. Using completion events adds extra overhead to the RNIC's PCIe bus. This overhead can be reduced by using selective signaling. When using a selectively signaled send queue of size $S$, up to $S-1$ consecutive verbs can be unsignaled, i.e., a completion event will not be pushed for these verbs. The receive queue cannot be selectively signaled. As $S$ is large ( 128), we use the terms "selective signaling" and "unsignaled" interchangeably.

\subsubsection{Transport types}

RDMA transports can be connected or unconnected. A connected transport requires a connection between two queue pairs that communicate exclusively with each other. Current RDMA implementations support two main types of connected transports: Reliable Connection (RC) and Unreliable Connection (UC). There is no acknowledgement of packet reception in UC; packets can be lost and the affected message can be dropped. As UC does not generate ACK/NAK packets, it causes less network traffic than RC.

In an unconnected transport, one queue pair can communicate with any number of other queue pairs. Current implementations provide only one unconnected transport: Unreliable Datagram (UD). The RNIC maintains state for each active queue in its queue pair context cache, so datagram transport can scale better for applications with a one-to-many topology.

InfiniBand and RoCE employ lossless link-level flow control, namely, credit-based flow control and Priority Flow Control. Even with unreliable transports (UC/UD), packets are never lost due to buffer overflows. Reasons for packet loss include bit errors on the wire and hardware failures, which are extremely rare. Therefore, our design, similar to choices made by Facebook and others [22], sacrifices transport-level retransmission for fast common case performance at the cost of rare application-level retries.

Some transport types support only a subset of the available verbs. Table 1 lists the verbs supported by each transport type. Figure 1 shows the DMA and network steps involved in posting verbs.

\subsection{Existing RDMA-based key-value stores}

Pilaf [21] is a key-value store that aims for high performance and low CPU use. For GETs, clients access a cuckoo hash table at the server using READs, which requires 2.6 round trips on average for single GET request. For PUTs, clients send their requests to the server using a SEND message. To ensure consistent GETs in the presence

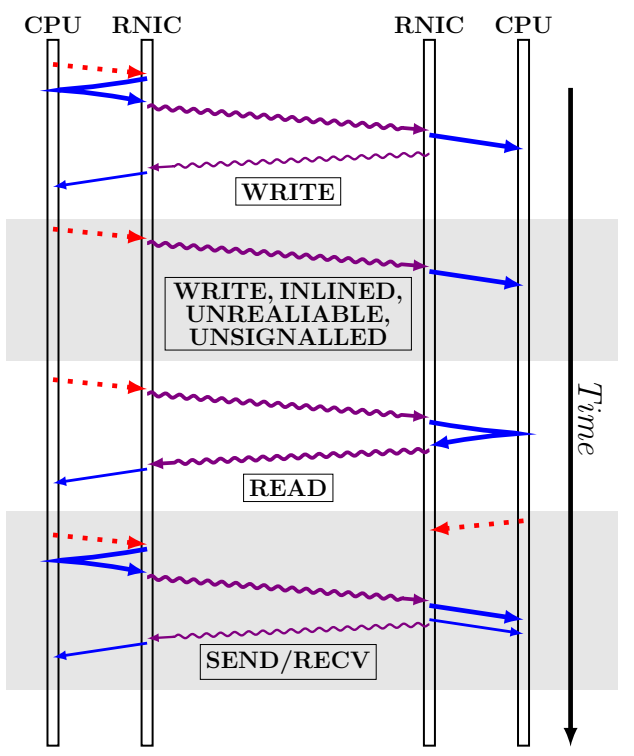

Figure 1: Steps involved in posting verbs. The dotted arrows are PCIe PIO operations. The solid, straight arrows are DMA operations: the thin ones are for writing the completion events. The thick wavy arrows are RDMA data packets and the thin ones are ACKs.

of concurrent PUTs, Pilaf's data structures are self-verifying: each hash table entry is augmented with two 64-bit checksums.

The second key-value store we compare against is based upon the store designed in FaRM [8]. It is important to note that FaRM is a more general-purpose distributed computing platform that exposes memory of a cluster of machines as a shared address space; we compare only against a key-value store implemented on top of FaRM that we call FaRM-KV. Unlike the client-server design in Pilaf and HERD, FaRM is symmetric, befitting its design as a cluster architecture: each machine acts as both a server and client.

FaRM's design provides two components for comparison. First is its key-value store design, which uses a variant of Hopscotch hashing [12] to create a locality-aware hash table. For GETs, clients READ several consecutive Hopscotch slots, one of which contains the key with high probability. Another READ is required to fetch the value if it is not stored inside the hash table. For PUTs, clients WRITE their request to a circular buffer in the server's memory. The server polls this buffer to detect new requests. This design is not specific to FaRM - we use it merely as an extant alternative to Pilaf's Cuckoo-based design to provide a more in-depth comparison for HERD.

The second important aspect of FaRM is its symmetry; here it differs from both Pilaf and HERD. For small, fixed-size key-value pairs, FaRM can "inline" the value with the key. With inlining, FaRM's RDMA read-based design still achieves lower maximum throughput than HERD, but it uses less CPU. This tradeoff may be right for a cluster where all machines are also busy doing computation; we do not evaluate the symmetric use case here, but it is an important consideration for users of either design.

\section{DESIGN DECISIONS}

Towards our goal of supporting key-value servers that achieve the highest possible throughput with RDMA, we explain in this section the reasons we choose to use-and not use-particular RDMA features and other design options. To begin with, we present an analysis of the performance of the RDMA verbs; we then craft a 


\begin{tabular}{lll} 
Name & Nodes & Hardware \\
\hline Apt & 187 & $\begin{array}{l}\text { Intel Xeon E5-2450 CPUs. ConnectX-3 } \\
\text { MX354A (56 Gbps IB) via PCIe 3.0 x8 }\end{array}$ \\
Susitna & 36 & $\begin{array}{l}\text { AMD Opteron 6272 CPUs. CX-3 MX353A } \\
\text { (40 Gbps IB) and CX-3 MX313A (40 Gbps } \\
\end{array}$ \\
& & RoCE) via PCIe 2.0 x8
\end{tabular}

Table 2: Cluster configuration

communication architecture using the fastest among them that can support our application needs.

As hinted in Section 1, one of the core decisions to make is whether to use memory verbs (RDMA read and write) or messaging verbs (SEND and RECV). Recent work from the systems and networking communities, for example, has focused on RDMA reads as a building block, because they bypass the remote network stack and CPU entirely for GETs [21, 8]. In contrast, however, the HPC community has made wider use of messaging, both for key-value caches [14] and general communication [16]. These latter systems scaled to thousands of machines, but provided low throughput-less than one million operations per second in memcached [14]. The reason for low throughput in [14] is not clear, but we suspect application design that makes the system incapable of leveraging the full power of the RNICs.

There remains an important gap between these two lines of work, and to our knowledge, HERD is the first system to provide the best of both worlds: throughput even higher than that of the RDMA-based systems while scaling to several hundred clients.

HERD takes a hybrid approach, using both RDMA and messaging to best effect. RDMA reads, however, are unattractive because of the need for multiple round trips. In HERD, clients instead write their requests to the server using RDMA writes over an Unreliable Connection (UC). This write places the PUT or GET request into a per-client memory region in the server. The server polls these regions for new requests. Upon receiving one, the server process executes in conventional fashion using its local data structures. It then sends a reply to the client using messaging verbs: a SEND over an Unreliable Datagram.

To explain why we use this hybrid of RDMA and messaging, we describe the performance experiments and analysis that support it. Particularly, we describe why we prefer using RDMA writes instead of reads, not taking advantage of hardware retransmission by opting for unreliable transports, and using messaging verbs despite conventional wisdom that they are slower than RDMA.

\subsection{Notation and experimental setup}

In the rest of this paper, we refer to an RDMA read as READ and to an RDMA write as WRITE. In this section, we present microbenchmarks from Emulab's [29] Apt cluster, a large, modern testbed equipped with 56 Gbps InfiniBand. Because Apt has only InfiniBand, in Section 5, we also use the NSF PRObE's [11] Susitna cluster to evaluate on RoCE. The hardware configurations of these clusters are shown in Table 2.

These experiments use one server machine and several client machines. We denote the server machine by $M_{S}$ and its RNIC by $R N I C_{S}$. Client machine $i$ is denoted by $C_{i}$. The server and client machines may run multiple server and client processes respectively. We call a message from client to server a request, and the reply from server to client, a response. The host issuing a verb is the requester and the destination host responder. For unsignaled SEND and WRITE over UC, the destination host does not actually send a response, but we still call it a responder.

For throughput experiments, processes maintain a window of several outstanding verbs in their send queues. Using windows allows us to saturate our RNICs with fewer processes. In all of our throughput experiments, we manually tune the window size for maximum aggregate throughput.

\subsection{Using WRITE instead of READ}

There are several benefits to using WRITE instead of READ. WRITEs can be performed over the UC transport, which itself confers several performance advantages. Because the responder does not need to send packets back, its RNIC performs less processing, and thus can support higher throughput than with READs. The reduced network bandwidth similarly benefits both the server and client throughput. Finally, as one might expect, the latency of an unsignaled WRITE is about half that ( $\frac{1}{2}$ RTT) of a READ. This makes it possible to replace one READ by two WRITEs, one client-to-server and one server-to-client (forming an applicationlevel request-reply pair), without increasing latency significantly.

\subsubsection{WRITEs have lower latency than READs}

Measuring the latency of an unsignaled WRITE is not straightforward as the requester gets no indication of completion. Therefore, we measure it indirectly by measuring the latency of an $E C H O$. In an ECHO, a client transmits a message to a server and the server relays the same message back to the client. If the ECHO is realized by using unsignaled WRITEs, the latency of an unsignaled WRITE is at most one half of the ECHO's latency.

We also measure the latency of signaled READ and WRITE operations. As these operations are signaled, we use the completion event to measure latency. For WRITE, we also measure the latency with payload inlining.

Figure 2 shows the average latency from these measurements. We use inlined and unsignaled WRITEs for ECHOs. On our RNICs, the maximum size of the inlined payload is 256 bytes. Therefore, the graphs for WR-INLINE and ECHO are only shown up to 256 bytes.

Unsignaled verbs: For payloads up to 64 bytes, the latency of ECHOs is close to READ latency, which confirms that the oneway WRITE latency is about half of the READ latency. For larger ECHOs, the latency increases because of the time spent in writing to the RNIC via PIO.

Signaled verbs: The solid lines in Figure 2 show the latencies for three signaled verbs-WRITE, READ, and WRITE with inlining (WR-INLINE). The latencies for READ and WRITE are similar because the length of the network/PCIe path travelled is identical. By avoiding one DMA operation, inlining reduces the latency of small WRITEs significantly.

\subsubsection{WRITEs have higher throughput than READs}

To evaluate throughput, it is first necessary to observe that with many client machines communicating with one server, different verbs perform very differently when used at the clients (talking to one server) and at the server (talking to many clients).

Inbound throughput: First, we measured the throughput for inbound verbs, i.e., the number of verbs that multiple remote machines (the clients) can issue to one machine (the server). Using the notation introduced above, $C_{1}, \ldots, C_{N}$ issue operations to $M_{S}$ as shown in Figure 3 a. Figure $3 b$ shows the cumulative throughput observed across the active machines. For up to 128 byte payloads, WRITEs achieve 35 Mops, which is about 34\% higher higher than 


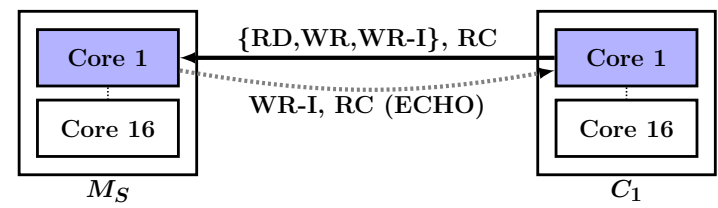

(a) Setup for measuring verbs and ECHO latency. We use one client process to issue operations to one server process

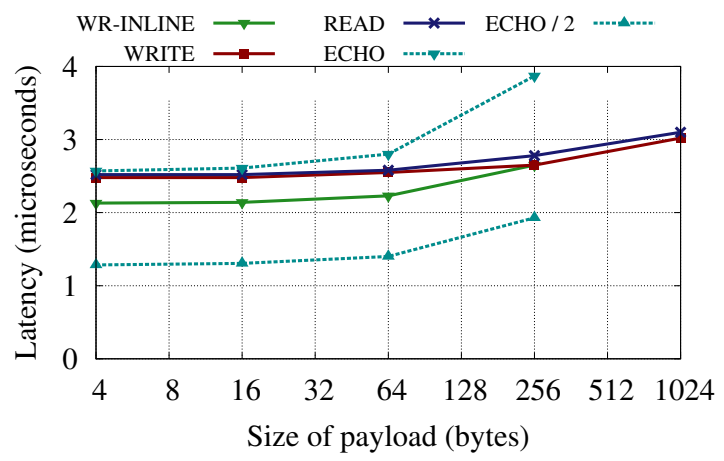

(b) The one-way latency of WRITE is half of the ECHO latency. ECHO operations used unsignaled verbs.

Figure 2: Latency of verbs and ECHO operations

the maximum READ throughput (26 Mops). Interestingly, reliable WRITEs deliver significantly higher throughput than READs despite their identical InfiniBand path. This is explained as follows: writes require less state maintainance both at the RDMA and the PCIe level because the initiator does not need to wait for a response. For reads, however, the request must be maintained in the initiator's memory till a response arrives. At the RDMA level, each queue pair can only service a few outstanding READ requests (16 in our RNICs). Similarly, at the PCIe level, reads are performed using non-posted transactions, whereas writes use cheaper, posted transactions.

Although the inbound throughput of WRITEs over UC and RC is nearly identical, using UC is still beneficial: It requires less processing at $R N I C_{S}$, and HERD uses this saved capacity to SEND responses.

Outbound throughput: We next measured the throughput for outbound verbs. Here, $M_{S}$ issues operations to $C_{1}, \ldots, C_{N}$. As shown in Figure $4 \mathrm{a}$, there are $N$ processes on $M_{S}$; the $i^{\text {th }}$ process communicates with $C_{i}$ only (the scalability problems associated with all-to-all communication are explained in Section 3.3). Apart from READs, WRITEs, and inlined WRITEs over UC, we also measure the throughput for inlined SENDs over UD for reasons outlined in Section 3.3. Figure $4 \mathrm{~b}$ plots the throughput achieved by $M_{S}$ for different payload sizes. For small sizes, inlined WRITEs and SENDs have significantly higher outbound throughput than READs. For large sizes, the throughput of all WRITE and SEND variants is less than for READs, but it is never less than $50 \%$ of the READ throughput. Thus, even for these larger items, using a single WRITE (or SEND) for responses remains a better choice than using multiple READs for key-value items.

ECHO throughput is interesting for two reasons. First, it provides an upper bound on the throughput of a key-value cache based on one round trip of communication. Second, ECHOs help characterize the processing power of the RNIC: although the advertised message rate of ConnectX-3 cards is 35 Mops, bidirectionally, they can process many more messages.

An ECHO consists of a request message and a response message. Varying the verbs and transport types yield several different imple-

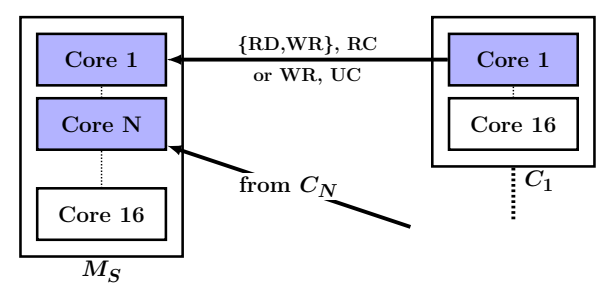

(a) Setup for measuring inbound throughput. Each client process communicates with only one server process

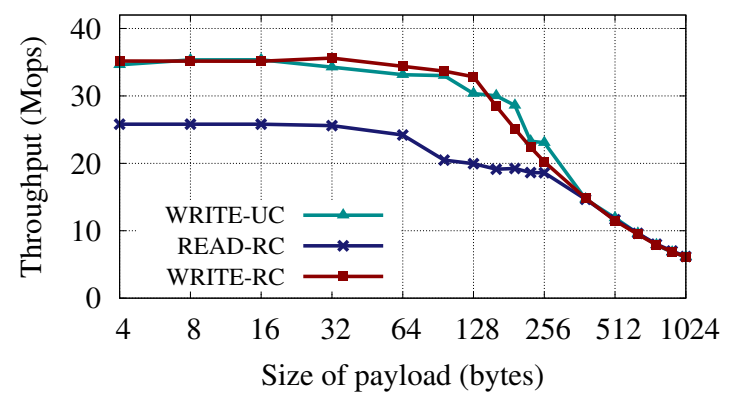

(b) For moderately sized payloads, WRITE has much higher inbound throughput than READs.

Figure 3: Comparison of inbound verbs throughput

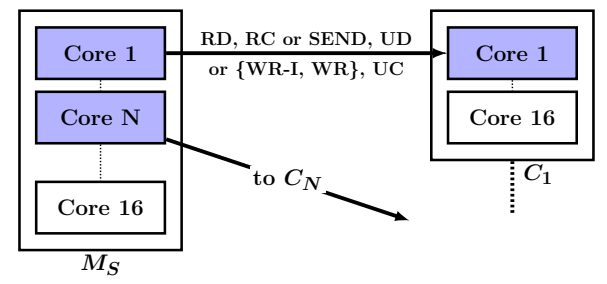

(a) Setup for measuring outbound throughput. Each server process communicates with only one client process.

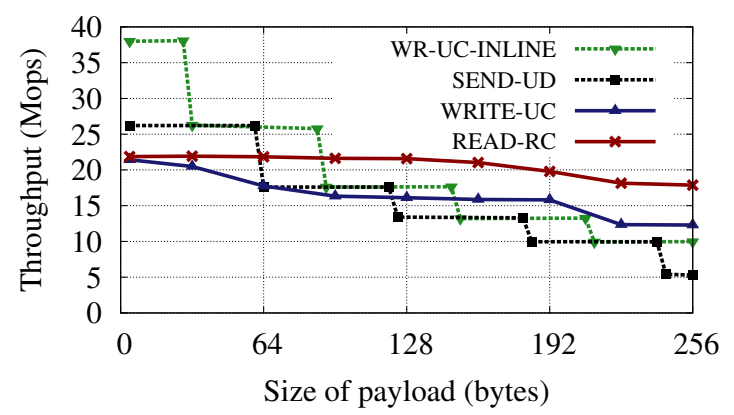

(b) For small payloads, WRITE with inlining has a higher outbound throughput than READ.

Figure 4: Comparison of outbound verbs throughput

mentations of ECHO. Figure 5 shows the throughput for some of the possible combinations and for 32 byte payloads. The figure also shows that using inlinining, selective signaling, and UC transport increases the performance significantly.

ECHOs achieve maximum throughput (26 Mops) when both the request and the response are done as RDMA writes. However, as shown in Section 3.3, this approach does not scale with the number of connections. HERD uses RDMA writes (over UC) for requests and SENDs (over UD) for responses. An ECHO server using this hybrid also achieves 26 Mops-it gives the performance of WRITEbased ECHOs, but with much better scalability. 


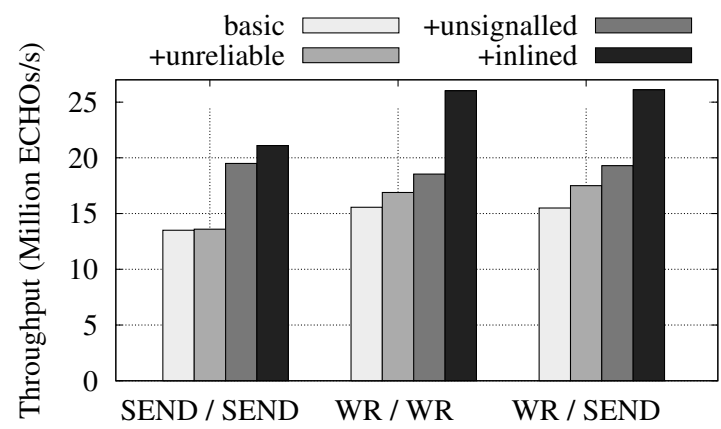

Figure 5: Throughput of ECHOs with 32 byte messages. In WR-SEND, the response is sent over UD.

By avoiding the overhead of posting RECVs at the server, our method of WRITE based requests and SEND-based responses provides better throughput than purely SEND-based ECHOs. Interestingly, however, after enabling all optimizations, the throughput of purely SEND-based ECHOs (with no RDMA operations) is 21 Mops, which is more than three-fourths of the peak inbound READ throughput (26 Mops). Both Pilaf and FaRM have noted that RDMA reads vastly outperform SEND-based ECHOs, which our results agree with if our optimizations are removed. With these optimizations, however, SENDs significantly outperform READs in cases where a single SEND-based ECHO can be used in place of multiple READs per request.

Our experiments show that several ECHO designs, with varying degrees of scalability, can perform better than multiple-READ designs. From a network-centric perspective, this is fortunate: it also means that designs that use only one cross-datacenter RTT can potentially outperform multiple-RTT designs both in throughput and in latency.

Discussion of verbs throughput: The ConnectX-3 card is advertised to support 35 million messages per second. Our experiments show that the card can achieve this rate for inbound WRITEs (Figure $3 b$ ) and slightly exceed it for very small outbound WRITEs (Figure 4b). All other verbs are slower than 30 Mops regardless of operation size. While the manufacturer does not specify bidirectional message throughput, we know empirically that $R N I C_{S}$ can service 30 million ECHOs per second (WRITE-based ECHOs achieve 30 Mops with 16 byte payloads; Figure 5 uses 32 byte payloads), or at least 60 total Mops of inbound WRITEs and outbound SENDs.

The reduced throughputs can be attributed to several factors:

- For outbound WRITEs larger than 28 bytes, the RNIC's message rate is limited by the PCIe PIO throughput. The sharp decreases in the WR-UC-INLINE and SEND-UD graphs in Figure $4 \mathrm{~b}$ at 64 byte intervals are explained by the use of write-combining buffers for PIO acceleration. With the writecombining optimization, the unit of PIO writes is a cache line instead of a word. Due to the larger datagram header, the throughput for SEND-UD drops for smaller payload sizes than for WRITEs

- The maximum throughput for inbound and outbound READs is 26 Mops and 22 Mops respectively, which is considerably smaller than the advertised 35 Mops message rate. Unlike WRITEs, READs are bottlenecked by the RNIC's processing power. This is as expected. Outbound READs involve a PIO operation, a packet transmission, a packet reception, and a DMA write, whereas outbound WRITEs (inlined and over UC) avoid the last two steps. Inbound READs require a DMA read by the RNIC followed by a packet transmission, whereas inbound WRITEs only require a DMA write.

\subsection{Using UD for responses}

Our previous experiments did not show that as the number of connections increases, connected transports begin to slow down. To reduce hardware cost, power consumption, and design complexity, RNICs have very little on-chip memory (SRAM) to cache address translation tables and queue pair contexts [26]. A miss in this cache requires a PCIe transaction to fetch the data from host memory. When the communication fan-in or fan-out exceeds the capacity of this cache, performance begins to suffer. This is a potentially important effect to avoid both for cluster scaling, but also because it interacts with the cache or store architectural decisions. For example, the cache design we build on in HERD partitions the keyspace between several server processes in order to achieve efficient CPU and memory utilization. Such partitioning further increases the fan-in and fan out of connections to a single machine.

To evaluate this effect, we modified our throughput experiments to enable all-to-all communication. We use $N$ client processes (one process each at $C_{1}, \ldots, C_{N}$ ) and $N$ server processes at $M_{S}$. For measuring inbound throughput, client processes select a server process at random and issue a WRITE to it. For outbound throughput, a server process selects a client at random and issues a WRITE to it. The results of these experiments for 32 byte messages are presented in Figure 6. Several results stand out:

Outbound WRITEs scale poorly: for $N=16$, there are 256 active queue pairs at $R N I C_{S}$ and the server-to-clients throughput degrades to $21 \%$ of the maximum outbound WRITE throughput (Figure $4 b$ ). With many active queue pairs, each posted verb can cause a cache miss, severely degrading performance.

Inbound WRITEs scale well: Clients-to-server throughput is high even for $N=16$. The reason for this is that queueing of outstanding verbs operations is performed at the requesting RNIC and very little state is maintained at the responding RNIC. Therefore, the responding RNIC can support a much larger number of active queue pairs without incurring cache misses. The higher requester overhead is amortized because the clients outnumber the server.

In a different experiment, we used 1600 client processes spread over 16 machines to issue WRITEs over UC to one server process. HERD uses this many-to-one configuration to reduce the number of active connections at the server (Section 4.2). This configuration also achieves 30 Mops.

Outbound WRITEs scale poorly only because $R N I C_{S}$ must manage many connected queue pairs. This problem cannot be solved if we use connected transports (RC/UC/XRC) because they require at least as many queue pairs at $M_{S}$ as the number of client machines. Scaling outbound communication therefore mandates using datagrams. UD transport supports one-to-many communication, i.e., a single UD queue can be used to issue operations to multiple remote UD queues. The main problem with using UD in a high performance application is that it only supports messaging verbs and not RDMA verbs.

Fortunately, messaging verbs only impose high overhead at the receiver. Senders can directly transmit their requests; only the receiver must pre-post a RECV before the SEND can be handled. For the sender, the work done to issue a SEND is identical to that required to post a WRITE. Figure 6 shows that, when performed over Unreliable Datagram transport, SEND side throughput is high and scales well with the number of connected clients. 


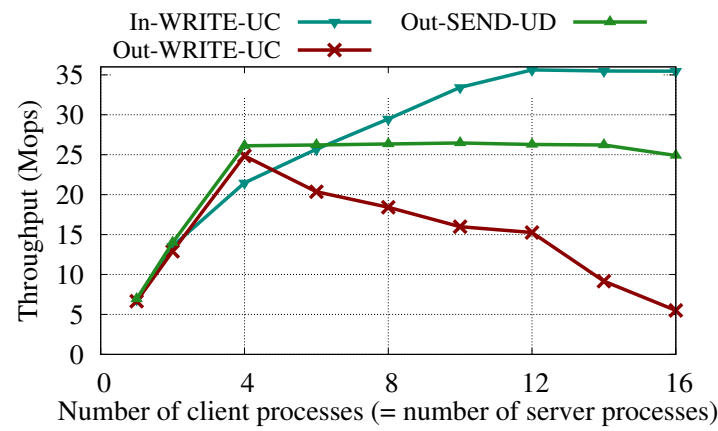

Figure 6: Comparison of UD and UC for all-to-all communication with 32 byte payloads. Inbound WRITEs over UC and outbound SENDs over UD scale well up to 256 queue pairs. Outbound WRITEs over UC scale poorly. All operations are inlined and unsignaled.

The slight degradation of SEND throughput beyond 10 connected clients happens because the SENDs are unsignaled, i.e., server processes get no indication of verb completion. This leads to the server processes overwhelming $R N I C_{S}$ with too many outstanding operations, causing cache misses inside the RNIC. As HERD uses SENDs for responding to requests, it can use new requests as an indication of the completion of old SENDs, thereby avoiding this problem.

\section{DESIGN OF HERD}

To evaluate whether these network-driven architectural decisions work for a real key-value application, we designed and implemented an RDMA-based KV cache, called HERD, based upon recent highperformance key-value designs. Our HERD setup consists of one server machine and several client machines. The server machine runs $N_{S}$ server processes. $N_{C}$ client processes are uniformly spread across the client machines.

\subsection{Key-Value cache}

The fundamental goal of this work is to evaluate our networking and architectural decisions in the context of key-value systems. We do not focus on building better back-end key-value data structures but rather borrow existing designs from MICA [18].

MICA is a near line-rate key-value cache and store for classical Ethernet. We restrict our discussion of MICA to its cache mode. MICA uses a lossy index to map keys to pointers, and stores the actual values in a circular log. On insertion, items can be evicted from the index (thereby making the index lossy), or from the log in a FIFO order. In HERD, each server process creates an index for $64 \mathrm{Mi}$ keys, and a 4 GB circular log. We use MICA's algorithm for both GETs and PUTs: each GET requires up to two random memory lookups, and each PUT requires one.

MICA shards the key space into several partitions based on a keyhash. In its "EREW" mode, each server core has exclusive read and write access to one partition. MICA uses the Flow Director [3] feature of modern Ethernet NICs to direct request packets to the core responsible for the given key. HERD achieves the same effect by allocating per-core request memory at the server, and allowing clients to WRITE their requests directly to the appropriate core.

\subsubsection{Masking DRAM latency with prefetching}

To service a GET, a HERD server must perform two random memory lookups, prepare the SEND response (with the key's value inlined in the WQE), and then post the SEND verb using the post_send () function. The memory lookups and the post_send () function are the two main sources of latency at the server. Each random memory

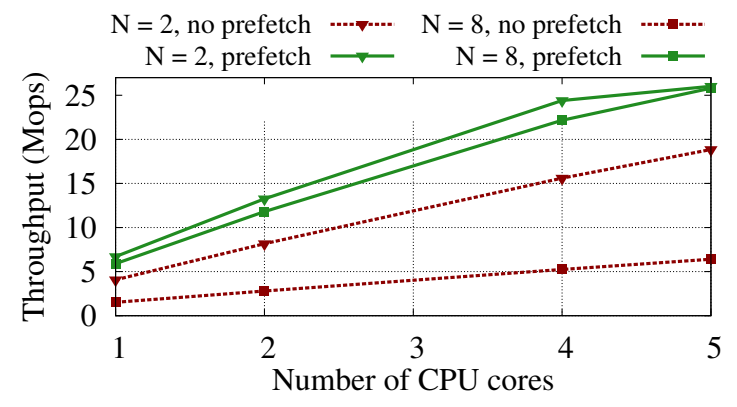

Figure 7: Effect of prefetching on throughput

access takes 60-120 ns and the post_send () function takes about $150 \mathrm{~ns}$. While the latter is unavoidable, we can mask the memory access latency by overlapping memory accesses of one request with computation of another request.

MICA and CuckooSwitch [18, 31] mask latency by overlapping memory fetches and prefetches, or request decoding and prefetches. HERD takes a different approach: we overlap prefetches with the post_send ( ) function used to transmit replies. To process multiple requests simultaneously in the absence of a driver that itself handles batches of packets $[2,18,31])$, HERD creates a pipeline of requests at the application level.

In HERD, the maximum number of memory lookups for each request is two. Therefore, we create a request pipeline with two stages. When a request is in stage $i$ of the pipeline, it performs the $i$-th memory access for the request and issues a prefetch for the next memory address. In this way, requests only access memory for which a prefetch has already been issued. On detecting a new request, the server issues a prefetch for the request's index bucket, advances the old requests in the pipeline, pushes in the new request, and finally calls post_send ( ) to SEND a reply for the pipeline's completed request. The server process expects the issued prefetches to finish by the time post_send () returns.

Figure 7 shows the effectiveness of prefetching. We use a WRITE/SEND-based ECHO server but this time the server performs $N$ random memory accesses before sending the response. Prefetching allows fewer cores to deliver higher throughput: 5 cores can deliver the peak throughput even with $N=8$. We conclude that there is significant headroom to implement more complex keyvalue applications, for instance, key-value stores, on top of HERD's request-reply communication mechanism.

With a large number of server processes, this pipelining scheme can lead to a deadlock. A server does not advance its pipeline until it receives a new request, and a client does not advance its request window until it gets a response. We avoid this deadlock as follows. While polling for new requests, if a server fails for 100 iterations consecutively, it pushes a no-op into the pipeline.

\subsection{Requests}

Clients WRITE their GET and PUT requests to a contiguous memory region on the server machine which is allocated during initialization. This memory region is called the request region and is shared among all the server processes by mapping it using shmget (). The request region is logically divided into $1 \mathrm{~KB}$ slots (the maximum size of a key-value item in HERD is $1 \mathrm{~KB}$ ).

Requests are formatted as follows. A GET request consists only of a 16-byte keyhash. A PUT request contains a 16-byte keyhash, a 2-byte LEN field (specifying the value's length), and up to 1000 bytes for the value. To poll for incoming requests, we use the left- 


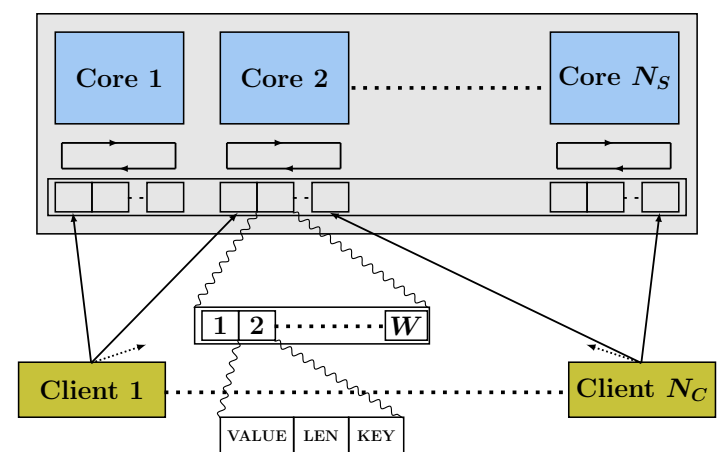

Figure 8: Layout of the request region at the server

to-right ordering of the RNIC's DMA writes [16, 8]. We use the keyhash field to poll for new requests; therefore, the key is written to the rightmost 16 bytes of the $1 \mathrm{~KB}$ slot. A non-zero keyhash indicates a new request, so we do not allow the clients to use a zero keyhash. The server zeroes out the keyhash field of the slot after sending a response, freeing it up for a new request.

Figure 8 shows the layout of the request region at the server machine. It consists of separate chunks for each server process which are further sub-divided into per-client chunks. Each perclient chunk consists of $W$ slots, i.e., each client can have up to $W$ pending requests to each server process. The size of the request region is $N_{S} \cdot N_{C} \cdot W \mathrm{~KB}$. With $N_{C}=200, N_{S}=16$ and $W=2$, this is approximately $6 \mathrm{MB}$ and fits inside the server's L3 cache. Each server process polls the per-client chunks for new requests in a round robin fashion. If server process $s$ has seen $r$ requests from client number $c$, it polls the request region at the request slot number $s \cdot\left(W \cdot N_{c}\right)+(c \cdot W)+r \bmod W$.

A network configuration using bidirectional, all-to-all, communication with connected transports would require $N_{C} \cdot N_{S}$ queue pairs at the server. HERD, however, uses connected transports for only the request side of communication, and thus requires only $N_{C}$ connected queue pairs. The configuration works as follows. An initializer process creates the request region, registers it with $R N I C_{S}$, establishes a UC connection with each client, and goes to sleep. The $N_{S}$ server processes then map the request region into their address space via shmget () and do not create any connections for receiving requests.

\subsection{Responses}

In HERD, responses are sent as SENDs over UD. Each client creates $N_{S}$ UD queue pairs (QPs) whereas each server process uses only one UD QP. Before writing a new request to server process $s$, a client posts a RECV to its $s$-th UD QP. This RECV specifies the memory area on the client where the server's response will be written. Each client allocates a response region containing $W \cdot N_{S}$ response slots: this region is used for the target addresses in the RECVs. After writing out $W$ requests, the client starts checking for responses by polling for RECV completions. On each successful completion, it posts another request.

The design outlined thus far deliberately shifts work from the server's RNIC to the client's, with the assumption that client machines often perform enough other work that saturating 40 or 56 gigabits of network bandwidth is not their primary concern. The servers, however, in an application such as Memcached, are often dedicated machines, and achieving high bandwidth is important.

\section{EVALUATION}

We evaluate HERD on two clusters: Apt and Susitna (Table 2). Due to limited space, we restrict our discussion to Apt and only present graphs for RoCE on Susitna. A detailed discussion of our results on Susitna may be found in [15]. Although Susitna uses similar RNICs as Apt, the slower PCIe 2.0 bus reduces the throughput of all compared systems. Despite this, our results on Susitna remain interesting: just as ConnectX-3 cards overwhelm PCIe $2.0 \mathrm{x} 8$, we expect the next-generation Connect-IB cards to overwhelm PCIe 3.0 $\mathrm{x} 16$. Our evaluation shows that:

- HERD uses the full processing power of the RNIC. A single HERD server can process up to 26 million requests per second. For value size up to 60 bytes, HERD's request throughput is greater than native READ throughput and is much greater than that of READ-based key-value services: it is over $2 \mathrm{X}$ higher than FaRM-KV and Pilaf.

- HERD delivers up to 26 Mops with approximately $5 \mu$ s average latency. Its latency is over $2 \mathrm{X}$ lower than Pilaf and FaRM-KV at their peak throughput respectively.

- HERD scales to the moderately sized Apt cluster, sustaining peak throughput with over 250 connected client processes.

We conclude the evaluation by examining the seeming drawback of the HERD design relative to READ-based designs-its higher server CPU use-and put this in context with the total (client + server) CPU required by all systems.

\subsection{Experimental setup}

We run all our throughput and latency experiments on 18 machines in Apt. The 17 client machines run up to 3 client processes each. With at most 4 outstanding requests per client, our implementation requires at least 36 client processes to saturate the server's throughput. We over-provision slightly by using 51 client processes. The server machine runs 6 server processes, each pinned to a distinct physical core. The machine configuration is described in Table 2. The machines run Ubuntu 12.04 with Mellanox's OFED v2.2 stack.

Comparison against stripped-down alternatives: In keeping with our focus on understanding the effects of network-related decisions, we compare our (full) HERD implementation against simplified implementations of Pilaf and FaRM-KV. These simplified implementations use the same communication methods as the originals, but omit the actual key-value storage, instead returning a result instantly. We made this decision for two reasons. First, while working with Pilaf's code, we observed several optimization opportunities; we did not want our evaluation to depend on the relative performance tuning of the systems. Second, we did not have access to the FaRM source code, and we could not run Windows Server on our cluster. Instead, we created and evaluated emulated versions of the two systems which do not include their backing data structures. This approach gives these systems the maximum performance advantage possible, so the throughput we report for both Pilaf and FaRM-KV may be higher than is actually achievable by those systems.

Pilaf is based on 2-level lookups: a hash-table maps keys to pointers. The pointer is used to find the value associated with the key from flat memory regions called extents. FaRM-KV, in its default operating mode, uses single-level lookups. It achieves this by inlining the value in the hash-table. It also has a two-level mode, where the value is stored "out-of-table." Because the out-of-table mode is necessary for memory efficiency with variable length keys, we compare HERD against both modes. In the following two subsections, we denote the size of a key, value, and pointer by $S_{K}, S_{V}$, and $S_{P}$ respectively. 


\subsubsection{Emulating Pilaf}

In $K$ - $B$ cuckoo hashing, every key can be found in $K$ different buckets, determined by $K$ orthogonal hash functions. For associativity, each bucket contains $B$ slots. Pilaf uses 3-1 cuckoo hashing with $75 \%$ memory efficiency and 1.6 average probes per GET (higher memory efficiency with fewer, but slightly larger, average probes is possible with 2-4 cuckoo hashing [9]). When reading the hash index via RDMA, the smallest unit that must be read is a bucket. A bucket in Pilaf has only one slot that contains a 4 byte pointer, two 8 byte checksums, and a few other fields. We assume the bucket size in Pilaf to be 32 bytes for alignment.

GET: A GET in Pilaf consists of 1.6 bucket READs (on average) to find the value pointer, followed by a $S_{V}$ byte READ to fetch the value. It is possible to reduce Pilaf's latency by issuing concurrent READs for both cuckoo buckets. As this comes at the cost of decreased throughput, we wait for the first READ to complete and issue the second READ only if it is required.

PUT: For a PUT, a client SENDs a $S_{K}+S_{V}$ byte message containing the new key-value item to the server. This request may require relocating entries in the cuckoo hash-table, but we ignore that as our evaluation focuses on the network communication only.

In emulating Pilaf, we enable all of our RDMA optimizations for both request types; we call the resulting system Pilaf-em-OPT.

\subsubsection{Emulating FaRM-KV}

FaRM-KV uses a variant of Hopscotch hashing to locate a key in approximately one READ. Its algorithm guarantees that a key-value pair is stored in a small neighborhood of the bucket that the key hashes to. The size of the neighborhood is tunable, but its authors set it to 6 to balance good space utilization and performance for items smaller than 128 bytes. FaRM-KV can inline the values in the buckets, or it can store them separately and only store pointers in the buckets. We call our version of FaRM-KV with inlined values FaRM-em and without inlining FaRM-em-VAR (for variable length values).

GET: A GET in FaRM-em requires a $6 *\left(S_{K}+S_{V}\right)$ byte READ. In FaRM-em-VAR, a GET requires a $6 *\left(S_{K}+S_{P}\right)$ byte READ followed by a $S_{V}$ byte READ.

PUT: FaRM-KV handles PUTs by sending messages to the server via WRITEs, similar to HERD. The server notifies the client of PUT completion using another WRITE. Therefore, a PUT in FaRM-em (and FaRM-em-VAR) consists of one $S_{K}+S_{V}$ byte WRITE from a client to the server, and one WRITE from the server to the client. For higher throughput, we perform these WRITEs over UC unlike the original FaRM paper that used RC (Figure 5).

\subsection{Workloads}

Three main workload parameters affect the throughput and latency of a key-value system: relative frequency of PUTs and GETs, item size, and skew.

We use two types of workloads: read-intensive (95\% GET, 5\% $\mathrm{PUT}$ ) and write-intensive (50\% GET, 50\% PUT). Our workload can either be uniform or skewed. Under a uniform workload, the keys are chosen uniformly at random from the 16 byte keyhash space. The skewed workload draws keys from a Zipf distribution with parameter .99. This workload is generated offline using YCSB [7]. We generated 480 million keys once and assigned 8 million keys to each of the 51 client processes.

\subsection{Throughput comparison}

We now compare the end-to-end throughput of HERD against the emulated versions of Pilaf and FaRM.

Figure 9 plots the throughput of these system for read-intensive and write-intensive workloads for 48-byte items $\left(S_{K}=16, S_{V}=32\right)$. We chose this item size because it is representative of real-life workloads: an analysis of Facebook's general-purpose key-value store [6] showed that the 50-th percentile of key sizes is approximately 30 bytes, and that of value sizes is 20 bytes. To compare the READbased GETs of Pilaf and FaRM with Pilaf's SEND/RECV-based PUTs, we also plot the throughput when the workload consists of $100 \%$ PUTs.

In HERD, both read-intensive and write-intensive workloads achieve 26 Mops, which is slightly larger than the throughput of native RDMA reads of a similar size (Figure 3b). For small keyvalue items, there is very little difference between PUT and GET requests at the RDMA layer because both types of requests fit inside one cacheline. Therefore, the throughput does not depend on the workload composition.

The GET throughput of Pilaf-em-OPT and FaRM-em(-VAR) is directly determined by the throughput of RDMA READs. A GET in Pilaf-em-OPT involves 2.6 READs (on average). Its GET throughput is 9.9 Mops, which is about $2.6 \mathrm{X}$ smaller than the maximum READ throughput. For GETs, FaRM-em requires a single 288 byte READ and delivers 17.2 Mops. FaRM-em-VAR requires a second READ and has throughput of 11.4 Mops for GETs.

Surprisingly, the PUT throughput in our emulated systems is much larger than their GET throughput. This is explained as follows. In FaRM-em(-VAR), PUTs use small WRITEs over UC that outperform the large READs required for GETs. Pilaf-em-OPT uses SEND/RECV-based requests and replies for PUT. Both Pilaf and FaRM assume that messaging-based ECHOs are much more expensive than READs. (Pilaf reports that for 17 byte messages, the throughput of RDMA reads is 2.449 Mops whereas the throughput of SEND/RECV-based ECHOs is only 0.668 Mops.) If SEND/RECV can provide only one fourth the throughput of READ, it makes sense to use multiple READs for GET.

However, we believe that these systems do not achieve the full capacity of SEND/RECV. After optimizing SENDs by using unreliable transport, payload inlining, and selective signaling, SEND/RECV based ECHOs, as shown in Figure 5, achieve 21 Mops, which is considerably more than half of our READ throughput (26 Mops). Therefore, we conclude that SEND/RECV-based communication, when used effectively, is more efficient than using multiple READs per request.

Figure 10 shows the throughput of the three systems with 16 byte keys and different value sizes for a read-intensive workload. For up to 60-byte items, HERD delivers over 26 Mops, which is slightly greater than the peak READ throughput. Up to 32-byte values, FaRM-em also delivers high throughput. However, its throughput declines quickly with increasing value size because the size of FaRMem's READs grow rapidly (as $\left.6 *\left(S_{V}+16\right)\right)$. This problem is fundamental to the Hopscotch-based KV design which amplifies the READ size to reduce round trips. FaRM-KV quickly saturates link bandwidths (PCIe or InfiniBand/RoCE) with smaller items than HERD, which conserves network bandwidth by transmitting only essential data. Figure 10 illustrates this effect. FaRM-em saturates the PCIe 2.0 bandwidth on Susitna with 4 byte values, and the 56 Gbps InfiniBand bandwidth on Apt with 32 byte values. HERD achieves high performance for up to 32 byte values on Susitna, and 


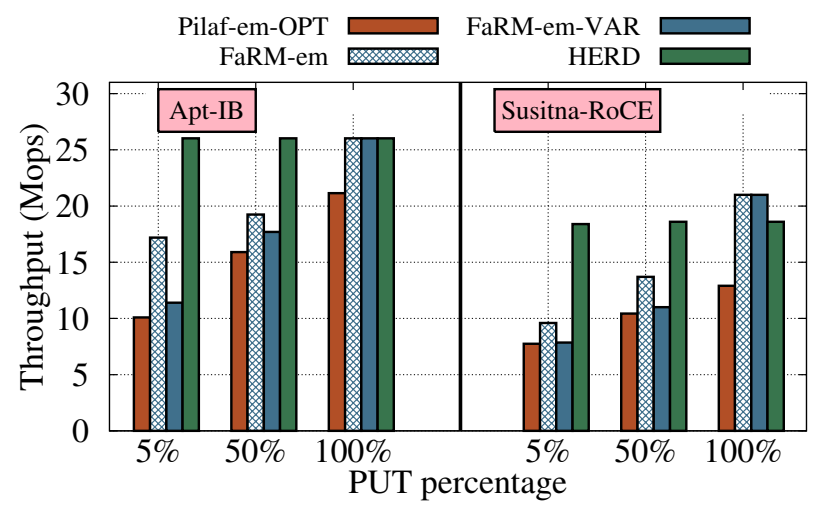

Figure 9: End-to-end throughput comparison for 48 byte key-value items
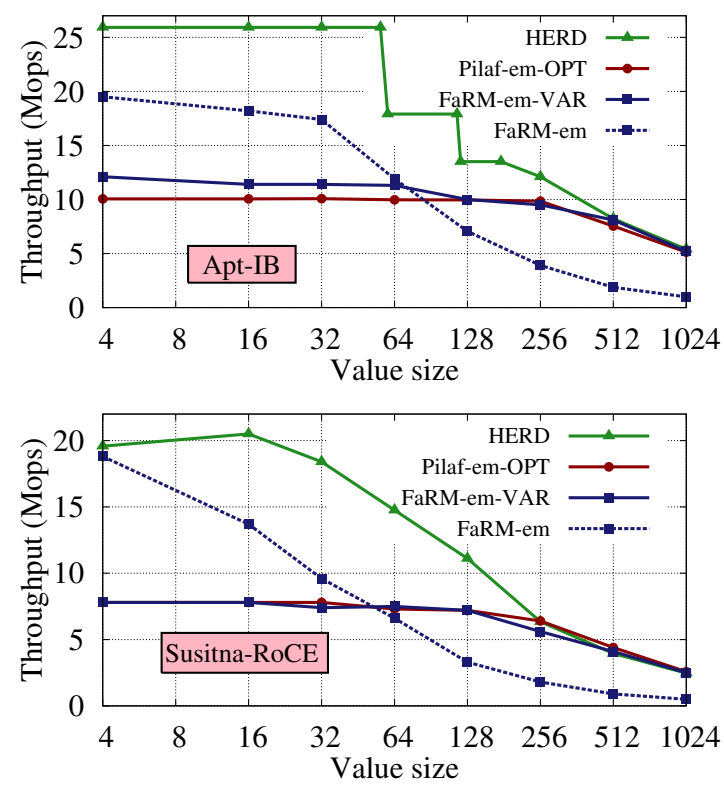

Figure 10: End-to-end throughput comparison with different value sizes

60 bytes values on Apt, and is bottlenecked by the smaller PCIe PIO bandwidth.

With large values (144 bytes on Apt, 192 on Susitna), HERD switches to using non-inlined SENDs for responses. The outbound throughput of large inlined messages is less than non-inlined messages because DMA outperforms PIO for large payloads (Figure 4b). For large values, the performance of HERD, FaRM-em, and Pilafem-OPT are within $10 \%$ of each other.

\subsection{Latency comparison}

Unlike FaRM-KV and Pilaf, HERD uses only one network round trip for any request. FaRM-KV and Pilaf use one round trip for PUT requests but require multiple round trips for GETs (except when FaRM-KV inlines values in the hash-table). This causes their GET latency to be higher than the latency of a single RDMA READ.

Figure 11 compares the average latencies of the three systems for a read-intensive workload; the error bars indicate the 5th and 95th percentile latency. To understand the dependency of latency on throughput, we increase the load on the server by adding more

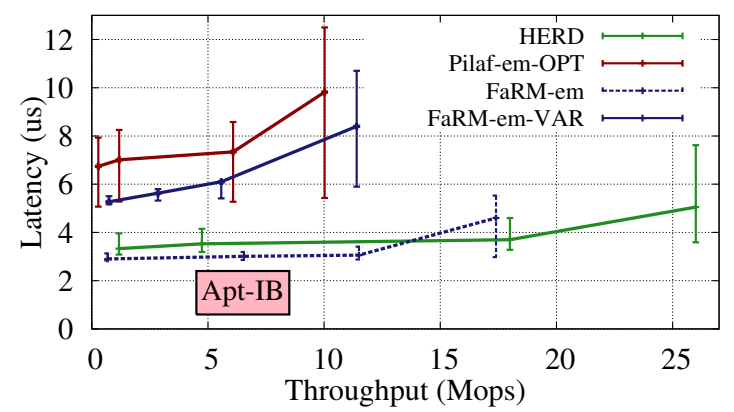

Figure 11: End-to-end latency with 48 byte items and read-intensive workload

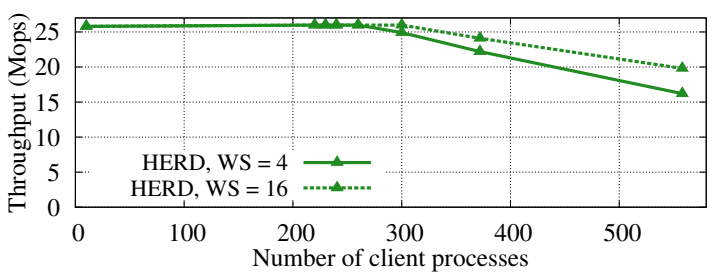

Figure 12: Throughput with variable number of client processes and different window sizes

clients until the server is saturated. When using 6 CPU cores at the server, HERD is able to deliver 26 million requests per second with approximately $5 \mu$ s average latency. For fixed-length keyvalue items, FaRM-em provides the lowest latency among the three systems because it requires only one network round trip (unlike Pilaf-em-OPT) and no computation at the server (unlike HERD). For variable length values, however, FaRM's variable length mode requires two RTTs, yielding worse latency than HERD.

The PUT latency for all three systems (not shown) is similar because the network path traversed is the same. The measured latency for HERD was slightly higher than that of the emulated systems because it performed actual hash table and memory manipulation for inserts, but this is an artifact of the performance advantage we give Pilaf-em and FaRM-em.

\subsection{Scalability}

We conducted a larger experiment to understand HERD's numberof-clients scalability. We used one machine to run 6 server processes and the remaining 186 machines for client processes. The experiment uses 16 byte keys and 32 byte values.

Figure 12 shows the results from this experiment. HERD delivers its maximum throughput for up to 260 client processes. With even more clients, HERD's throughput starts decreasing almost linearly. The rate of decrease can be reduced by increasing the number of outstanding requests maintained by each client, at the cost of higher request latency. Figure 12 shows the results for two window sizes: 4 (HERD's default) and 16. This observation suggests that the decline is due to cache misses in $R N I C_{S}$, as more outstanding verbs in a queue can reduce cache pressure. We expect this scalability limit to be resolved with the introduction of Dynamically Connected Transport in the new Connect-IB cards [1, 8],

Another likely scalability limit of our current HERD design is the round-robin polling at the server for requests. With thousands of clients, using WRITEs for inbound requests may incur too much CPU overhead; mitigating this effect may necessitate switching to a SEND/SEND architecture over Unreliable Datagram transport. 


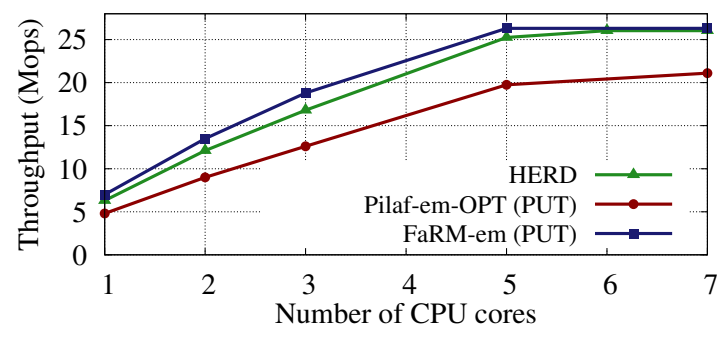

Figure 13: Throughput as a function of server CPU cores

Figure 5 shows there is a 4-5 Mops decrease to this change, but once made, the system should scale up to many thousands of clients, while still outperforming an RDMA READ-based architecture. ${ }^{1}$ We expect the performance of the SEND/SEND architecture relative to WRITE-SEND to increase with the introduction of inlined RECVs in Connect-IB cards. This will reduce the load on RNICs by encapsulating the RECV payload in the RECV completion.

\subsection{HERD CPU Use}

The primary drawback of not using READs in HERD is that GET operations require the server $\mathrm{CPU}$ to execute requests, in exchange for saving one cross-datacenter RTT. While at first glance, it might seem that HERD's CPU usage should be higher than Pilaf and FaRM$\mathrm{KV}$, we show that in practice these two systems also have significant sources of CPU usage that reduce the extent of the difference.

First, issuing extra READs adds CPU overhead at the Pilaf and FaRM-KV clients. To issue the second READ, the clients must poll for the first READ to complete. HERD shifts this overhead to the server's CPU, making more room for application processing at the clients.

Second, handling PUT requests requires $\mathrm{CPU}$ involvement at the server. Achieving low-latency PUTs requires dedicating server CPU cores that poll for incoming requests. Therefore, the exact CPU use depends on the fraction of PUT throughput that server is provisioned for, because this determines the CPU resources that must be allocated to it, not the dynamic amount actually used. For example, our experiments show that, even ignoring the cost of updating data structures, provisioning for 100\% PUT throughput in Pilaf and FaRM-KV requires over 5 CPU cores. Figure 13 shows FaRM-em and Pilaf-em-OPT's PUT throughput for 48 byte key-value items and different numbers of CPU cores at the server. Pilaf-em-OPT's CPU usage is higher because it must post RECVs for new PUT requests, which is more expensive than FaRM-em's request-region polling.

In Figure 13, we also plot HERD's throughput for the same workload by varying the number of server CPU cores. HERD is able to deliver over $95 \%$ of its maximum throughput with 5 CPU cores. The modest gap to FaRM-em arises because the HERD server in this experiment is handling hash table lookups and updates, whereas the emulated FaRM-KV is handling only the network traffic.

We believe, therefore, that HERD's higher throughput and lower latency, along with the significant CPU utilization in Pilaf and FaRM$\mathrm{KV}$, justifies the architectural decision to have the CPU involved on the GET path for small key-value items. For a 50\% PUT workload, for example, the moderate extra cost of adding a few more cores-or using the already-idle cycles on the cores-is likely worthwhile for many applications.

${ }^{1}$ Figure 5 uses SENDs over UC, but we have verified that similar throughput is possible using SENDs over UD.

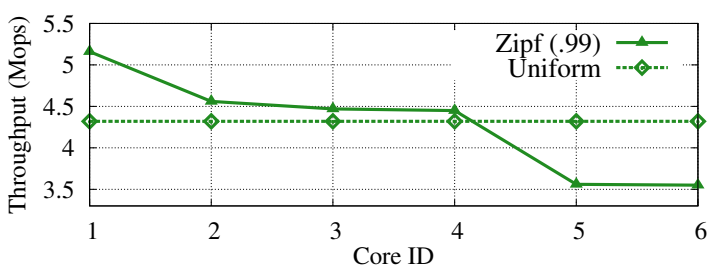

Figure 14: Per-core throughput under skewed and uniform workloads. Note that the $y$-axis does not begin at 0 .

\subsection{Resistance to skew}

To understand how HERD's behavior is impacted by skew, we tested it with a workload where the keys are drawn from a Zipf distribution. HERD adapts well to skew, delivering its maximum performance even when the Zipf parameter is .99. HERD's resistance to skew comes from two factors. First, the back-end MICA architecture [18] that we use in HERD performs well under skew; a skewed workload spread across several partitions produces little variation in the partitions' load compared to the skew in the workload's distribution. Under our Zipf-distributed workload, with 6 partitions, the most loaded CPU core is only $50 \%$ more so than the least loaded core, even though the most popular key is over $10^{5}$ times more popular than the average.

Second, because the CPU cores share the RNIC, the highly loaded cores are able to benefit from the idle time provided by the less-used cores. Figure 13 demonstrates this effect: with a uniform workload and using only a single core, HERD can deliver 6.3 Mops. When the system is configured to use 6 cores-the minimum required by HERD to deliver its peak throughput- the system delivers 4.32 Mops per core. The per-core performance reduction is not because of a CPU bottleneck, but because the server processes saturate the PCIe PIO throughput. Therefore, even if the workload is skewed, there is ample CPU headroom on a given core to handle the extra requests.

Figure 14 shows the per-core throughput of HERD for a skewed workload. The experimental configuration is: 48-byte items, readintensive, skewed workload, 6 total CPU cores. The per-core throughput for a uniform workload is included for comparison.

\section{RELATED WORK}

RDMA-based key-value stores: Other than Pilaf and FaRM, several projects have designed memcached-like systems over RDMA. Panda et al. [14] describe a memcached implementation using a hybrid of UD and RC transports. It uses SEND/RECV messages for all requests and avoids the overhead of UD transport (caused by a larger header size than RC) by actively switching connections between RC and UD. Although their cluster (ConnectX, 32 Gbps) is comparable to Susitna (ConnectX-3, $40 \mathrm{Gbps}$ ), their request rate is less than 1.5 Mops. Stuedi et al. [25] describe a SoftiWARP [28] based version of memcached targeting CPU savings in wimpy nodes with $10 \mathrm{GbE}$.

Accelerating systems with RDMA: Several projects have used verbs to improve the performance of systems such as HBase, Hadoop RPC, PVFS [30, 13, 20]. Most of these use only SEND/RECV verbs as a fast alternative to socket-based communication. In a PVFS implementation over InfiniBand [30], read ( ) and write () operations in the filesystem use both RDMA and SEND/RECV. They favor WRITEs over READs for the same reasons as in our work, suggesting that the performance gap has existed over several generations of InfiniBand hardware. There have been several versions 
of MPI over InfiniBand [16, 19]. MPICH2 uses RDMA writes for one-sided messaging: the server polls the head of a circular buffer that is written to by a client. HERD extends this messaging in a scalable fashion for all-to-all request-reply communication. While [30, 13, 20, 16, 19] have benchmarked verbs performance before, it has been for large messages in the context of applications like NFS and MPI. Our work exploits the performance differences that appear only for small messages and are relevant for message rate-bound applications like key-value stores.

User level networking: Taken together, we believe that one conclusion to draw from the union of HERD, Pilaf, FaRM, and MICA [18] is that the biggest boost to throughput comes from bypassing the network stack and avoiding CPU interrupts, not necessarily from bypassing the CPU entirely. All four of these systems use mechanisms to allow user-level programs to directly receive requests or packets from the NIC: the userlevel RDMA drivers for HERD, Pilaf, and FaRM, and the Intel DPDK library for MICA. As we discuss below, the throughput of these systems is similar, but the batching required by the DPDK-based systems confers a latency advantage to the hardware-supported InfiniBand systems. These lessons suggest profitable future work in making user-level classical Ethernet systems more portable, easier to use, and lower-latency. One ongoing effort is NIQ [10], an FPGA-based low-latency NIC which uses cacheline-sized PIOs (without any DMA) to transmit and receive small packets. Inlined WRITEs in RDMA use the same mechanism at the requesters's side.

General key-value stores: MICA [18] is a recent key-value system for classical Ethernet. It assigns exclusive partitions to server cores to minimize memory contention, and exploits the NIC's capability to steer requests to the responsible core [3]. A MICA server delivers 77 Mops with 4 dual-port, 10 Gbps PCIe 2.0 NICs, with $50 \mu$ s average latency (19.25 Gbps with one PCIe 2.0 card). This suggests that, comparing the state-of-the-art, classical Ethernet-based solutions can provide comparable throughput to RDMA-based solutions, although with much higher latency. RAMCloud [23] is a RAM-based, persistent key-value store that uses messaging verbs for low latency communication.

\section{CONCLUSION}

This paper explored the options for implementing fast, low-latency key-value systems atop RDMA, arriving at an unexpected and novel combination that outperforms prior designs and uses fewer network round-trips. Our work shows that, contrary to widely held beliefs about engineering for RDMA, single-RTT designs with server CPU involvement can outperform the "optimization" of CPU-bypassing remote memory access when the RDMA approaches require multiple RTTs. These results contribute not just a practical artifact- the HERD low-latency, high-performance key-value cache-but an improved understanding of how to use RDMA to construct future DRAM-based storage services.

Acknowledgements We thank our shepherd Chuanxiong Guo and the anonymous reviewers for their feedback that helped improve the paper. We also thank Miguel Castro and Dushyanth Narayanan for discussing FaRM with us, Kirk Webb and Robert Ricci for getting us early access to the Apt cluster, and Hyeontaek Lim for his valuable insights. This work was supported by funding from the National Science Foundation under awards CNS-1314721 and CCF-0964474, and Intel via the Intel Science and Technology Center for Cloud Computing (ISTC-CC). The PRObE cluster [11] used for many experiments is supported in part by NSF awards CNS-1042537 and CNS-1042543 (PRObE).

\section{References}

[1] Connect-IB: Architecture for Scalable High Performance Computing. URL http://www.mellanox.com/relateddocs/applications/SB_Connect-IB.pdf.

[2] Intel DPDK: Data Plane Development Kit. URL http: //dpdk . org.

[3] Intel 8259910 Gigabit Ethernet Controller: Datasheet. URL http://www. intel.com/content/www/us/en/ethernetcontrollers/82599-10-gbe-controller-datasheet.html.

[4] Redis: An Advanced Key-Value Store. URL http://redis.io.

[5] memcached: A Distributed Memory Object Caching System, 2011. URL http://memcached.org.

[6] B. Atikoglu, Y. Xu, E. Frachtenberg, S. Jiang, and M. Paleczny. Workload Analysis of a Large-Scale Key-Value Store. In SIGMETRICS, 2012.

[7] B. F. Cooper, A. Silberstein, E. Tam, R. Ramakrishnan, and R. Sears. Benchmarking Cloud Serving Systems with YCSB. In SoCC, 2010.

[8] A. Dragojevic, D. Narayanan, O. Hodson, and M. Castro. FaRM: Fast Remote Memory. In USENIX NSDI, 2014

[9] B. Fan, D. G. Andersen, and M. Kaminsky. MemC3: Compact and Concurrent MemCache with Dumber Caching and Smarter Hashing. In USENIX NSDI, 2013.

[10] M. Flajslik and M. Rosenblum. Network Interface Design for Low Latency Request-Response Protocols. In USENIX ATC, 2013.

[11] G. Gibson, G. Grider, A. Jacobson, and W. Lloyd. PRObE: A Thousand-Node Experimental Cluster for Computer Systems Research.

[12] M. Herlihy, N. Shavit, and M. Tzafrir. Hopscotch Hashing. In DISC, 2008.

[13] J. Huang, X. Ouyang, J. Jose, M. W. ur Rahman, H. Wang, M. Luo, H. Subramoni, C. Murthy, and D. K. Panda. High-Performance Design of HBase with RDMA over InfiniBand. In IPDPS, 2012.

[14] J. Jose, H. Subramoni, K. C. Kandalla, M. W. ur Rahman, H. Wang, S. Narravula, and D. K. Panda. Scalable Memcached Design for InfiniBand Clusters Using Hybrid Transports. In CCGRID. IEEE, 2012.

[15] A. Kalia, D. G. Andersen, and M. Kaminsky. Using RDMA Efficiently for Key-Value Services. In Technical Report CMU-PDL-14-106, 2014.

[16] J. Li, J. Wu, and D. K. Panda. High Performance RDMA-Based MPI Implementation over InfiniBand. International Journal of Parallel Programming, 2004.

[17] H. Lim, B. Fan, D. G. Andersen, and M. Kaminsky. SILT: A Memory-efficient, High-performance Key-value Store. In SOSP, 2011.

[18] H. Lim, D. Han, D. G. Andersen, and M. Kaminsky. MICA: A Holistic Approach to Fast In-Memory Key-Value Storage. In USENIX NSDI, 2014.

[19] J. Liu, W. Jiang, P. Wyckoff, D. K. Panda, D. Ashton, D. Buntinas, W. Gropp, and B. Toonen. Design and Implementation of MPICH2 over InfiniBand with RDMA Support. In IPDPD, 2004.

[20] X. Lu, N. S. Islam, M. W. ur Rahman, J. Jose, H. Subramoni, H. Wang, and D. K. Panda. High-Performance Design of Hadoop RPC with RDMA over InfiniBand. In $I C P P, 2013$.

[21] C. Mitchell, Y. Geng, and J. Li. Using One-Sided RDMA Reads to Build a Fast, CPU-Efficient Key-Value Store. In USENIX ATC, 2013.

[22] R. Nishtala, H. Fugal, S. Grimm, M. Kwiatkowski, H. Lee, H. C. Li, R. McElroy, M. Paleczny, D. Peek, P. Saab, D. Stafford, T. Tung, and V. Venkataramani. Scaling Memcache at Facebook. In USENIX NSDI, 2013.

[23] D. Ongaro, S. M. Rumble, R. Stutsman, J. Ousterhout, and M. Rosenblum. Fas Crash Recovery in RAMCloud. In SOSP, 2011.

[24] R. Pagh and F. F. Rodler. Cuckoo Hashing. J. Algorithms, 2004.

[25] P. Stuedi, A. Trivedi, and B. Metzler. Wimpy Nodes with 10GbE: Leveraging One-Sided Operations in Soft-RDMA to Boost Memcached. In USENIX ATC, 2012.

[26] S. Sur, A. Vishnu, H.-W. Jin, W. Huang, and D. K. Panda. Can Memory-Less Network Adapters Benefit Next-Generation InfiniBand Systems? In HOTI, 2005.

[27] S. Sur, M. J. Koop, L. Chai, and D. K. Panda. Performance Analysis and Evaluation of Mellanox ConnectX Infiniband Architecture with Multi-Core Platforms. In HOTI, 2007.

[28] A. Trivedi, B. Metzler, and P. Stuedi. A Case for RDMA in Clouds: Turning Supercomputer Networking into Commodity. In APSys, 2011.

[29] B. White, J. Lepreau, L. Stoller, R. Ricci, S. Guruprasad, M. Newbold, M. Hibler, C. Barb, and A. Joglekar. An Integrated Experimental Environment for Distributed Systems and Networks. In OSDI, 2002.

[30] J. Wu, P. Wyckoff, and D. K. Panda. PVFS over InfiniBand: Design and Performance Evaluation. In Ohio State University Tech Report, 2003.

[31] D. Zhou, B. Fan, H. Lim, M. Kaminsky, and D. G. Andersen. Scalable, High Performance Ethernet Forwarding with CuckooSwitch. In CoNEXT, 2013. 